\title{
Clinical outcomes of the sequential use of pazopanib followed by everolimus for the treatment of metastatic renal cell carcinoma: a multicenter study in Korea
}

\author{
Jeong Ho Kim, $\mathrm{MD}^{1}$; Wan Lee, MD, $\mathrm{PhD}^{1}$; Tae Nam Kim, $\mathrm{MD}^{2}$; Jong Kil Nam, MD, $\mathrm{PhD}^{2}$; \\ Tae Hyo Kim, $\mathrm{MD}^{3}$; Ki Soo Lee, $\mathrm{MD}^{3}$ \\ ${ }^{1}$ Dongnam Institute of Radiological \& Medical Sciences Cancer Centre; ${ }^{2}$ Department of \\ Urology, Pusan National University Hospital, Busan; ${ }^{3}$ Department of Urology, Dong-A \\ University Hospital, Busan; Korea
}

Cite as: Can Urol Assoc J 2017 Dec. 1; Epub ahead of print. http://dx.doi.org/10.5489/cuaj.4644

\section{Published online December 1, 2017}

$* * *$

\section{Abstract}

Introduction: The aim of this study was to investigate the real-world clinical outcomes of first-line pazopanib and second-line everolimus in Korean patients with metastatic renal cell carcinoma (mRCC).

Methods: Data of patients who had mRCC with clear-cell component between 2001 and 2015 at multiple institutions were collected retrospectively. To be included in the analysis, patients had to meet the following criteria: age $\geq 18$ years; received first-line targeted therapy with pazopanib; and received second-line targeted therapy with everolimus. The primary outcomes included overall survival (OS), progression-free survival (PFS), and adverse events (AEs).

Results: A total of 36 patients were included in the analysis. The median followup period was 33.5 months (range 17-49.5). The median PFS was eight months (95\% confidence interval [CI] 6.4-9.6) after treatment with pazopanib and three months (95\% CI 1.9-4.1) with everolimus. The median OS was 27 months (95\% CI 16.6-37.4). The median treatment duration was seven months (range 4.3-10.8) after treatment with pazopanib and 3.5 months (range 3-4) with everolimus. Multivariate analysis revealed that the Heng risk criteria were independently associated with OS $(\mathrm{p}<0.001)$. Almost every patient experienced some form of $\mathrm{AE}$, the majority of which were mostly mild or moderate in severity. The most common AEs were diarrhea (50\%), hypertension (44.4\%), and fatigue (41.7\%) after treatment with pazopanib, and anemia (47.2\%), stomatitis (41.7\%), and fatigue (38.9\%) with everolimus. Conclusions: The outcomes for the patients treated with pazopanib followed by everolimus in Korea as observed by us were consistent with those reported by previous studies. The Heng risk criteria were significantly associated with the prognosis of patients with mRCC. AEs were mainly mild to moderate and readily managed. 


\section{Introduction}

During the last decade, a number of novel agents that have markedly improved the prognosis of patients with metastatic renal cell carcinoma (mRCC) have been introduced in clinical practice. ${ }^{1,2}$ These agents work by targeting angiogenesis through pathways involving the vascular endothelial growth factor (VEGF) receptor and mammalian target of rapamycin (mTOR). One of these agents, pazopanib, is an oral tyrosine kinase inhibitor (TKI) that targets the VEGF receptor, platelet-derived growth factor (PDGF) receptor, and c-kit, and is approved as first-line treatment for mRCC. ${ }^{3}$ Pazopanib has been shown to prolong progression-free survival (PFS) compared to placebo in patients with mRCC. ${ }^{4}$ In the large phase III COMPARZ (Comparing the efficacy, safety, and tolerability of pazopanib versus sunitinib) trial, pazopanib showed similar efficacy to sunitinib, which is currently the most widely used first-line drug for the treatment of mRCC. ${ }^{5}$ The present guidelines recommend the use of pazopanib as first-line treatment for favorable- or intermediate- risk patients with clear cell mRCC.

The phase III study of everolimus in patients with mRCC whose disease had progressed despite receiving VEGF targeted therapy demonstrated that patients treated with everolimus had a better PFS compared with those treated with placebo. ${ }^{6,7}$ Adverse events were more frequent in the everolimus group, but were mostly of mild or moderate severity. ${ }^{6,7}$ Everolimus was the first mTOR inhibitor to be approved for sequential use after a prior targeted therapy, and it is one of the most commonly used treatments in this setting. ${ }^{8-10}$ For the second-line treatment of mRCC, several targeted agents have been introduced, including TKIs and mTOR inhibitors; however, in Korea, everolimus is the only available drug for VEGF-refractory patients with mRCC. Although several studies have proposed an optimal sequence of targeted therapy, no firm recommendations are currently available. ${ }^{11}$

The pivotal trial for these two agents has provided clinicians and patients with promising results, but few studies have examined the efficacy and tolerability of these drugs in Asian patients. ${ }^{3,6}$ Therefore, this study aimed to assess the efficacy and safety of pazopanib and everolimus in Korean patients with mRCC.

\section{Methods}

\section{Patients and methods}

Clinical data of patients treated with pazopanib and everolimus for mRCC were retrospectively collected between 2001 and 2015 at four different institutions (Dongnam Institute of Radiological \& Medical Sciences Cancer Center, Dong-A University hospital, Pusan National University Hospital, Pusan National University Yangsan Hospital) from the medical record system. The inclusion criteria were as follows: age $\geq 18$ years; histologically confirmed clear-cell RCC; metastases measurable on computed tomography (CT) or magnetic resonance imaging (MRI); a performance status of 0-2 based on Eastern Cooperative Oncology Group (ECOG) criteria; and receiving first-line treatment with pazopanib and second-line treatment with everolimus. After excluding patients aged $<18$ 
years and those who received prior chemotherapy or cytokines, 36 patients were enrolled. Baseline demographics and clinicopathological data were collected. The treatment continued until disease progression or unacceptable toxicity. The response was measured using the Response Evaluation Criteria in Solid Tumors (RECIST) version 1.0. Adverse events (AEs) were evaluated according to Common Terminology Criteria for Adverse Events (CTCAE) version 3.0. The primary end-point of this study was PFS. PFS was defined as the time from the initiation of treatment to the date of progressive disease or death. Overall survival (OS) was defined as the time from the initiation of treatment to all-cause death.

This study was approved by the Institutional Review Board of Dongnam Institute of Radiological \& Medical Sciences Cancer Center and performed according to the ethical standard laid down by the 1964 declaration of Helsinki and its later amendments. As this was a retrospective study, the requirement of informed consent was waived.

\section{Statistical analysis}

OS and PFS were calculated using the Kaplan-Meier method, and 95\% confidence interval (CI) was considered statistical significant. All statistical analyses were performed using IBM SPSS v.20 software (SPSS, Inc., Chicago, IL), with a two-sided $p$ value $<0.05$ indicating statistical significance.

\section{Results}

The clinicopathological characteristics of the 36 patients included in this study are summarized in Table 1. Of these patients, 5 patients (16.7\%) had favorable risk disease, 26 patients $(72.7 \%)$ had intermediate risk disease, and 4 patients $(11.1 \%)$ had poor risk disease according to the Heng risk criteria. ${ }^{12}$ Nephrectomy was performed in 21 patients (58.3\%). The rest of the patients underwent ultrasound-guided percutaneous renal biopsy and diagnosed with RCC.

\section{First-line pazopanib}

The median PFS with first-line pazopanib was 8 months (95\% CI, 6.64-9.36). Of the 36 patients, 12 (33.3\%) achieved a partial response, 11 (30.6\%) had stable disease, and 13 (36.1\%) had progressive disease, as the best response (Table 2). The median treatment duration was 7 months (95\% CI, 6.37-10.98). The primary reason for treatment discontinuation was disease progression (88.9\%), followed by AEs (11.1\%). Most patients (77.8\%) experienced AEs of any grade (Table 3). The most common AEs were diarrhea (50\%), hypertension (44.4\%), fatigue (41.7\%), nausea (33.3\%), and vomiting (33.3\%). The proportion of patients experiencing an AE with maximum grade of $3 / 4$ was $50 \%$. The most common laboratory abnormalities were serum creatinine elevation (19.4\%), aspartate aminotransferase elevation (16.7\%), and anemia (13.9\%).

\section{Second-line everolimus}

The median PFS with second-line everolimus was 3 months (95\% CI, 2.08-3.92). Of the 36 patients, 3 (8.3\%) achieved a partial response, 17 (47.2\%) had stable disease, and 13 (36.1\%) had progressive disease, as best response (Table 2). The median treatment duration was 3.5 
months (95\% CI, 3.14-4.86). The primary reason for treatment discontinuation was disease progression (66.7\%), followed by AEs (22.2\%). Most patients (94.8\%) experienced AEs of any grade (Table 3). The most common AEs were stomatitis (41.7\%), fatigue (38.9\%), rash (36.1\%), asthenia (27.8\%), and anorexia (25\%). The most common laboratory abnormalities were anemia (47.2\%), serum cholesterol elevation (38.9\%), and serum glucose elevation (30.1\%). No statistical association was found between the therapeutic effects of pazopanib in the first-line therapy and those of everolimus.

\section{Overall survival}

The median OS of the 36 patients analyzed in the study was 27 months (95\% CI, 16.6-37.4 months) (Figure 1). Multivariate analysis revealed that the Heng risk criteria were independently associated with OS $(p<0.001)$ (Table 4).

\section{Discussion}

In the phase III pivotal trial, pazopanib showed promising anti-tumor activity in patients with mRCC. Pazopanib significantly prolonged PFS compared with placebo in the treatment-naïve subpopulation (11.1 months in the pivotal trial and 8.4 months in the COMPARZ trial)., Several retrospective studies reported similar efficacy. For example, Matrana et al. investigated the efficacy of pazopanib as a first-line setting for mRCC and reported a median OS of 29.1 months and a median PFS of 13.7 months. ${ }^{13}$ In another study on the efficacy of pazopanib, Vogelzang et al. reported that the median OS and PFS were 22 months and 8.5 months, respectively. ${ }^{14}$ In a study of Asian patients, Kim et al. reported that the median OS and PFS were 21.9 months and 12.2 months, respectively. ${ }^{15}$ In our study, the median PFS for patients treated with first-line pazopanib was 8 months, which was slightly shorter compared to the studies mentioned above.

The efficacy of everolimus in mRCC is well known, but there are subtle differences in PFS. Several studies on everolimus in patients with VEGF-refractory mRCC reported a median PFS ranging from 3.8 months to 6.9 months. ${ }^{6,16-20}$ Almost all of these studies have demonstrated a slightly better outcome than that obtained in this study. There are several reasons for these differences in outcome. First, the proportion of patients with favorable risk in this study was relatively low compared with other studies. The importance of prognostic models was well defined and widely used to predict the results of VEGF-targeted therapy. ${ }^{12,21}$ According to the above-mentioned reports, the hazards ratio for OS increased from favorable risk criteria to poor risk criteria. Second, the number of patients who had undergone nephrectomy was low in this study. The nephrectomy rate was $58.3 \%$ in the present study, and this is relatively low compared to that in other studies in which the nephrectomy rate was 80 96\%. ${ }^{3,6,15-20}$ The benefit of cytoreductive nephrectomy is not well defined in the era of targeted therapy; however, many studies showed improved OS in patients treated with cytoreductive surgery. ${ }^{22-24}$ In the present study, nephrectomy was not universally performed because this decisions was left to the discretion of the surgeon. Third, this study included Asian patients, and therefore, it is possible that outcomes were affected by ethnic differences between study populations. Although the COMPARZ trial reported no significant differences 
across ethnic groups in the results of pazopanib's clinical activity and tolerability profile, reliable data on ethnic differences in the efficacy of everolimus are not yet available. ${ }^{5}$ Therefore, we think that these discrepancies in patient characteristics may have adversely affected outcomes in our patients compared to those reported in other studies. The patients included in this study slightly differ from those enrolled in clinical trials, and may be more similar to the patient group observed by clinicians in daily practice. Because of this, we believe that our results are more representative of real-world outcomes.

The multivariate analysis of PFS revealed that the Heng risk criteria were significantly related with prognosis. Currently, the most widely used prognostic models for mRCC are the Memorial Sloan-Kettering Cancer Center (MSKCC) and the Heng model. According to Kwon et al., the Heng model had slightly better discriminatory ability than the MSKCC model for Korean patients. ${ }^{21}$ Therefore, the present study applied the Heng model to classify each patient.

There is no consensus regarding the best sequential use of targeted therapies. ${ }^{8,25,26}$ Although guidelines recommend initial treatment with VEGF targeted therapy for most patients with mRCC, subsequent treatment options have not been clearly defined. Everolimus has been widely accepted as the most appropriate second-line treatment for mRCC to date, and it is the only available second-line agent in Korea. However, nivolumab and cabozantinib showed superior efficacy compared with everolimus in recent studies, and therefore guidelines have recommended these agents for VEGF-refractory mRCC. ${ }^{16,20,27}$ Given the ongoing research and clinical updates, it is likely that the treatment paradigms are going to change in the near future.

This study has some limitations. First, this is a retrospective study; therefore, we could not exclude possible selection bias. Second, because of the small sample size, caution is needed when generalizing the study results. Third, there was no common follow-up protocol between the institutions included in this study, and this could have affected the study results. Fourth, the goal of this study was to observe the response of patients following drug administration in a real clinical setting. It was not to compare the therapeutic effects of different drugs. Thus, it must be noted during the interpretation of our study results that the therapeutic effects of the drugs discussed in this study cannot be objectively compared to those of other drugs.

\section{Conclusion}

Although this study showed slightly different results compared with the previously cited clinical trials, we found that pazopanib and everolimus were efficacious in the treatment of mRCC and were well tolerated in Korean patients. The prognosis of mRCC was significantly associated with the Heng risk criteria. Further investigations determining the optimal treatment algorithm should be conducted to ensure patients obtain the maximum possible benefit from their treatment. 


\section{References}

1. Figlin R, Sternberg C, Wood CG. Novel agents and approaches for advanced renal cell carcinoma. J Urol 2012;188:707-15.

2. Hwang E, Lee HJ, Sul CK, et al. Efficacy and safety of sunitinib on metastatic renal cell carcinoma: a single-institution experience. Korean J Urol 2010;51:450-5.

3. Sternberg CN, Davis ID, Mardiak J, et al. Pazopanib in locally advanced or metastatic renal cell carcinoma: results of a randomized phase III trial. J Clin Oncol 2010;28:1061-8. 4. Sternberg CN, Hawkins RE, Wagstaff J, et al. A randomised, double-blind phase III study of pazopanib in patients with advanced and/or metastatic renal cell carcinoma: final overall survival results and safety update. Eur J Cancer 2013;49:1287-96.

5. Motzer RJ, Hutson TE, Cella D, et al. Pazopanib versus sunitinib in metastatic renal-cell carcinoma. N Engl J Med 2013;369:722-31.

6. Motzer RJ, Escudier B, Oudard S, et al. Efficacy of everolimus in advanced renal cell carcinoma: a double-blind, randomised, placebo-controlled phase III trial. Lancet 2008;372:449-56.

7. Motzer RJ, Alyasova A, Ye D, et al. Phase II trial of second-line everolimus in patients with metastatic renal cell carcinoma (RECORD-4). Ann Oncol 2016;27:441-8.

8. Heng DY, Signorovitch J, Swallow E, et al. Comparative Effectiveness of Second-Line Targeted Therapies for Metastatic Renal Cell Carcinoma: A Systematic Review and MetaAnalysis of Real-World Observational Studies. PLoS One 2014;9:e114264.

9. Di Lorenzo G, Autorino R, Sternberg CN. Metastatic renal cell carcinoma: recent advances in the targeted therapy era. Eur Urol 2009;56:959-71.

10. Jonasch E, Signorovitch JE, Lin PL, et al. Treatment patterns in metastatic renal cell carcinoma: a retrospective review of medical records from US community oncology practices. Curr Med Res Opin 2014;30:2041-50.

11. Ljungberg B, Bensalah K, Canfield S, et al. EAU guidelines on renal cell carcinoma: 2014 update. Eur Urol 2015;67:913-24.

12. Heng DY, Xie W, Regan MM, et al. Prognostic factors for overall survival in patients with metastatic renal cell carcinoma treated with vascular endothelial growth factor-targeted agents: results from a large, multicenter study. J Clin Oncol 2009;27:5794-9.

13. Matrana MR, Bathala T, Campbell MT, et al. Outcomes of unselected patients with metastatic clear-cell renal cell carcinoma treated with first-line pazopanib therapy followed by vascular endothelial growth factor receptor tyrosine kinase inhibitors or mammalian target of rapamycin inhibitors: a single institution experience. BJU Int 2016;118:264-71.

14. Vogelzang NJ, Hackshaw MD, Hutson TE, et al. First-Line and Sequential Use of Pazopanib Followed by Mammalian Target of Rapamycin Inhibitor Therapy Among Patients With Advanced Renal Cell Carcinoma in a US Community Oncology Setting. Clin Genitourin Cancer 2015;13:210-7.

15. Kim MJ, Park SH, Lee JL, et al. A Korean multi-center, real-world, retrospective study of 
first-line pazopanib in unselected patients with metastatic renal clear-cell carcinoma. $B M C$ Urol 2016;16:46.

16. Choueiri TK, Escudier B, Powles T, et al. Cabozantinib versus Everolimus in Advanced Renal-Cell Carcinoma. N Engl J Med 2015;373:1814-23.

17. Albiges L, Kube U, Eymard JC, et al. Everolimus for patients with metastatic renal cell carcinoma refractory to anti-VEGF therapy: results of a pooled analysis of non-interventional studies. Eur J Cancer 2015;51:2368-74.

18. Bergmann L, Kube U, Doehn C, et al. Everolimus in metastatic renal cell carcinoma after failure of initial anti-VEGF therapy: final results of a noninterventional study. BMC cancer 2015;15:303.

19. Patel SB, Stenehjem DD, Gill DM, et al. Everolimus Versus Temsirolimus in Metastatic Renal Cell Carcinoma After Progression With Previous Systemic Therapies. Clin Genitourin Cancer 2016;14:153-9.

20. Motzer RJ, Escudier B, McDermott DF, et al. Nivolumab versus Everolimus in Advanced Renal-Cell Carcinoma. N Engl J Med 2015;373:1803-13.

21. Kwon WA, Cho IC, Yu A, et al. Validation of the MSKCC and Heng risk criteria models for predicting survival in patients with metastatic renal cell carcinoma treated with sunitinib. Ann Surg Oncol 2013;20:4397-404.

22. Dabestani S, Marconi L, Hofmann F, et al. Local treatments for metastases of renal cell carcinoma: a systematic review. Lancet Oncology 2014;15:e549-e61.

23. Thomas AZ, Adibi M, Slack RS, et al. The Role of Metastasectomy in Patients with Renal Cell Carcinoma with Sarcomatoid Dedifferentiation: A Matched Controlled Analysis. J Urol 2016;196:678-84.

24. Patel HD, Gorin MA, Gupta N, et al. Mortality trends and the impact of lymphadenectomy on survival for renal cell carcinoma patients with distant metastasis. Can Urol Assoc J 2016;10:389-95.

25. Singh P, Agarwal N, Pal SK. Sequencing systemic therapies for metastatic kidney cancer. Curr Treat Options Oncol 2015;16:316.

26. Bedke J, Gauler T, Grünwald V, et al. Systemic therapy in metastatic renal cell carcinoma. World J Urol 2016.

27. Powles T, Staehler M, Ljungberg B, et al. Updated EAU Guidelines for Clear Cell Renal Cancer Patients Who Fail VEGF Targeted Therapy. Eur Urol 2016;69:4-6. 


\section{Figures and Tables}

Fig. 1. Kaplan-Meier survival curve of overall survival. (A) Median overall survival: 27 months (95\% CI 16.6-37.4 months); (B) overall survival stratified by Heng risk criteria.

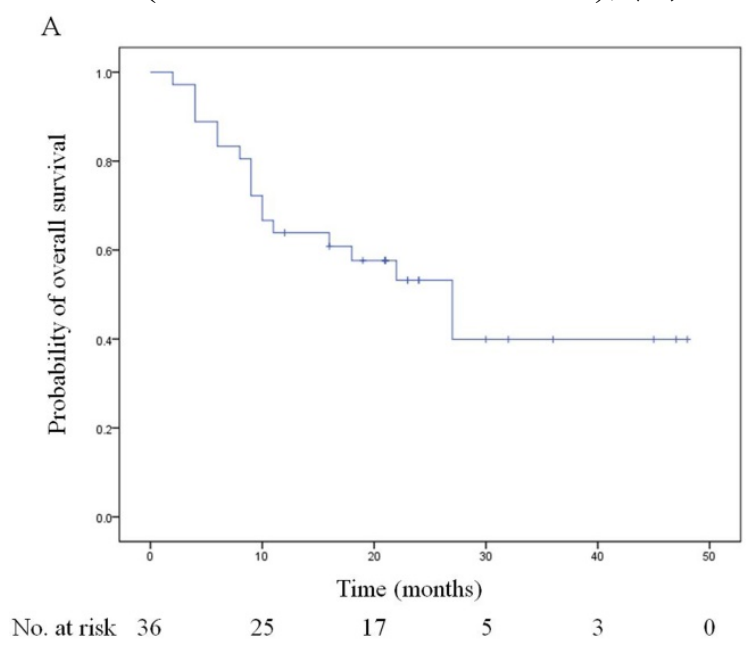

$\mathrm{B}$

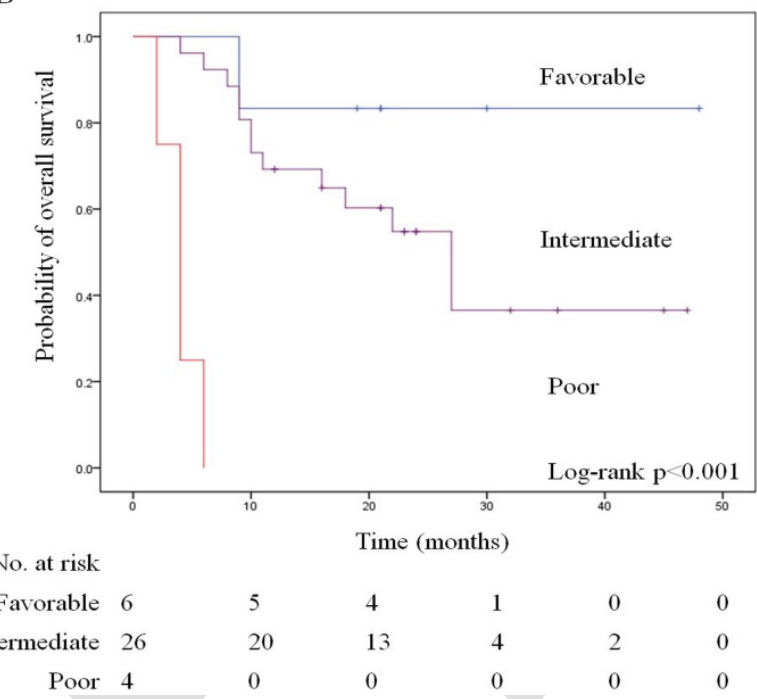

\begin{tabular}{|l|c|}
\hline Table 1. Patients characteristics & 36 \\
\hline Variables, $\mathrm{n}$ & $65.9 \pm 9.9$ \\
\hline Age (years) mean \pm SD & $28(77.8)$ \\
\hline Sex & $8(22.2)$ \\
\hline Male (\%) & $22.76 \pm 3.59$ \\
\hline Female (\%) & $33.5(17-49.5)$ \\
\hline BMI (kg/m ${ }^{2}$ ) mean \pm SD & $8(22.2)$ \\
\hline Followup period (months) median (range) & $18(50)$ \\
\hline DM (\%) & $2(5.6)$ \\
\hline Hypertension (\%) & $2(5.6)$ \\
\hline CKD (\%) & $13(36.1)$ \\
\hline Cardiovascular disease (\%) & $6(16.7)$ \\
\hline Symptoms (\%) & $26(72.7)$ \\
\hline Heng risk criteria & $4(11.1)$ \\
\hline Favourable (\%) & \\
\hline Intermediate (\%) & $21(58.3)$ \\
\hline Poor (\%) & \\
\hline Diagnostic methods & $27(75)$ \\
\hline Nephrectomy & \\
\hline Sites of metastatic disease & \\
\hline Lung & \\
\hline
\end{tabular}




\begin{tabular}{|c|c|}
\hline Liver & $6(16.7)$ \\
\hline Bone & $15(41.7)$ \\
\hline Brain & $2(5.6)$ \\
\hline
\end{tabular}

BMI: body mass index; CKD: chronic kidney disease; DM: diabetes mellitus;

SD: standard deviation.

\begin{tabular}{|l|c|}
\hline \multicolumn{2}{|l|}{ Table 2. Summary of efficacy } \\
\hline Variables, $\mathrm{n}$ & 36 \\
\hline Pazopanib & $0(0)$ \\
\hline Best response (\%) & $12(33.3)$ \\
\hline Complete response & $11(30.6)$ \\
\hline Partial response & $13(36.1)$ \\
\hline Stable disease & $8(6.4-9.6)$ \\
\hline Progressive disease & \\
\hline $\begin{array}{l}\text { Overall progression-free survival, months } \\
\text { (95\% CI) }\end{array}$ & \\
\hline Everolimus & $0(0)$ \\
\hline Best response (\%) & $3(8.3)$ \\
\hline Complete response & $17(47.2)$ \\
\hline Partial response & $13(36.1)$ \\
\hline Stable disease & $3(8.3)$ \\
\hline Progressive disease & $3(1.9-4.1)$ \\
\hline Unknown & $27(16.6-37.4)$ \\
\hline $\begin{array}{l}\text { Overall progression-free survival, months } \\
\text { (95\% CI) }\end{array}$ & \\
\hline Overall survival, months (95\% CI) & \\
\hline
\end{tabular}

CI: confidence interval. 
Table 3. Treatment-related adverse events

\begin{tabular}{|c|c|c|c|c|c|}
\hline \multicolumn{3}{|c|}{ Pazopanib $(n=36)$} & \multicolumn{3}{|c|}{ Everolimus $(n=36)$} \\
\hline & \multicolumn{2}{|c|}{ Grade } & & \multicolumn{2}{|c|}{ Grade } \\
\hline & Any & $\geq \mathbf{3}$ & & Any & $\geq \mathbf{3}$ \\
\hline Non-hematological & & & Non-hematological & & \\
\hline Diarrhea & $18(50)$ & $1(2.8)$ & Stomatitis & 15 (41.7) & $1(2.8)$ \\
\hline Hypertension & $16(44.4)$ & $0(0)$ & Fatigue & 14 (38.9) & $1(2.8)$ \\
\hline Fatigue & $15(41.7)$ & $1(2.8)$ & Rash & $13(36.1)$ & $0(0)$ \\
\hline Vomiting & $12(33.3)$ & $1(2.8)$ & Asthenia & $10(27.8)$ & $0(0)$ \\
\hline Nausea & $12(33.3)$ & $2(5.6)$ & Anorexia & $9(25)$ & $0(0)$ \\
\hline Asthenia & $11(30.1)$ & $0(0)$ & Nausea & $8(22.2)$ & $0(0)$ \\
\hline Hair color change & $11(30.1)$ & $0(0)$ & Diarrhea & $7(19.4)$ & $1(2.8)$ \\
\hline Anorexia & $10(27.8)$ & $0(0)$ & Pneumonitis & $4(11.1)$ & $2(5.6)$ \\
\hline Headache & $3(8.3)$ & $0(0)$ & Infection (Pneumonia) & $3(8.3)$ & $0(0)$ \\
\hline Peripheral edema & $2(5.6)$ & $0(0)$ & Vomiting & $2(5.6)$ & $0(0)$ \\
\hline Stomatitis & $1(2.8)$ & $0(0)$ & Peripheral edema & $2(5.6)$ & $0(0)$ \\
\hline Infection (pneumonia) & $1(2.8)$ & $0(0)$ & Epistaxis & $2(5.6)$ & $0(0)$ \\
\hline Hematological & & & Hematological & 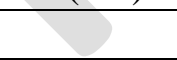 & \\
\hline $\begin{array}{l}\text { Decreased } \\
\text { hemoglobin }\end{array}$ & 5 (13.9) & $1(0)$ & $\begin{array}{l}\text { Decreased } \\
\text { hemoglobin }\end{array}$ & $17(47.2)$ & $2(2.8)$ \\
\hline Decreased neutrophil & $5(13.9)$ & $0(0)$ & Decreased leukocyte & $10(27.8)$ & $0(0)$ \\
\hline Decreased leukocyte & $3(8.3)$ & $0(0)$ & Thrombocytopenia & $9(25)$ & $0(0)$ \\
\hline Thrombocytopenia & $2(5.6)$ & $0(0)$ & Lymphocytopenia & $7(19.4)$ & $0(0)$ \\
\hline Clinical chemistry & 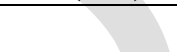 & & Clinical chemistry & & \\
\hline Increased creatinine & $7(19.4)$ & $0(0)$ & Increased cholesterol & $14(38.9)$ & $0(0)$ \\
\hline Increased AST & $6(16.7)$ & $1(2.8)$ & Increased glucose & $11(30.1)$ & $0(0)$ \\
\hline Hypocalcemia & $3(8.3)$ & $0(0)$ & Increased creatinine & $9(25)$ & $0(0)$ \\
\hline Hyponatremia & $3(8.3)$ & $0(0)$ & Increased AST & $7(19.4)$ & $1(2.8)$ \\
\hline Increased glucose & $2(5.6)$ & $0(0)$ & Hypophosphatemia & $6(16.7)$ & $0(0)$ \\
\hline Hypoglycemia & $2(5.6)$ & $0(0)$ & Increased ALT & $5(13.9)$ & $1(2.8)$ \\
\hline Increased cholesterol & $2(5.6)$ & $1(2.8)$ & Hyponatremia & $3(8.3)$ & $0(0)$ \\
\hline
\end{tabular}

AST: aspartate aminotransferase; ALT: alanine aminotransferase. 
Table 4. Univariate and multivariate Cox regression analyses of overall survival

\begin{tabular}{|l|c|c|c|}
\hline \multicolumn{1}{|c|}{ Variable } & $\begin{array}{c}\text { Univariate } \\
\text { analysis }\end{array}$ & \multicolumn{2}{|c|}{ Multivariate analysis } \\
\hline Heng risk criteria & $\mathbf{p}$ & $\mathbf{p}$ & $\begin{array}{c}\text { Adjusted HR } \\
\mathbf{( 9 5 \% ~ C I ) ~}\end{array}$ \\
\hline Intermediate & $<0.001$ & $<0.001$ & \\
\hline High & 0.229 & 0.229 & $3.49(0.46-26.69)$ \\
\hline Nephrectomy & 0.001 & 0.001 & $91.39(6.83-1223.14)$ \\
\hline No. of metastasis & 0.237 & 0.485 & $0.679(0.23-2.01)$ \\
\hline Synchronous metastasis & 0.857 & 0.752 & $0.89(0.44-1.8)$ \\
\hline Site of metastasis & 0.388 & 0.399 & $1.57(0.55-4.44)$ \\
\hline With bone metastasis & 0.424 & 0.494 & \\
\hline With brain metastasis & 0.247 & 0.278 & $1.87(0.6-5.78)$ \\
\hline
\end{tabular}

CI: confidence interval; HR: hazard ratio. 